\title{
Blended learning versus traditional teaching-learning-setting: Evaluation of cognitive and affective learning outcomes for the inter-professional field of occupational medicine and prevention
}

\section{Blended Learning versus traditionelles Lehr-Lernsetting: Evaluierung von kognitiven und affektiven Lernergebnissen für das interprofessionelle Arbeitsfeld Arbeitsmedizin und Prävention}

\author{
Ursula Eckler ${ }^{1 *}$, Andrea Greisberger ${ }^{1}$, \\ Franziska Höhne' ${ }^{1}$ Peter Putz \\ ${ }^{1}$ Fachhochschule Campus Wien, Health Sciences, \\ 1100 Wien, AUSTRIA \\ *ursula.eckler@fh-campuswien.ac.at
}

Received 29 March 2017, accepted 22 September 2017, available online 30 November 2017

\begin{abstract}
Blended learning is characterised as a combination of face-to-face teaching and e-learning in terms of knowledge transfer, students' learning activities and reduced presence at the teaching facility. The present cohort study investigated long-term effects of blended learning regarding cognitive outcomes as well as self-indicated estimates of immediate learning effects on the affective domain in the inter-professional field of occupational medicine. Physiotherapy students (bachelor degree) at FH Campus Wien - University of Applied Sciences completed the course Occupational Medicine/Prevention either in a traditional teaching-learning setting entirely taught face-to-face (control-group, $n=94$ ), or with a blended learning model (intervention-group, $n=93$ ). Long-term effects (1.5 year follow-up) on the cognitive learning outcomes were assessed according to four levels of Bloom's learning objectives. In addition, students estimated potential benefits resulting from blended learning based on four Krathwohl's learning objectives for the affective domain by means of a six-option Likert scale $(n=282)$. Concerning cognitive outcomes, significant results favouring both groups were found with effect sizes from small to medium. The traditional teaching-learning setting resulted in significantly better results in the upmost aspired learning objective (analysis) at the long-term $(p<0,01 ; r=-0,33)$. In contrast, the intervention group resulted in significantly better long-term results on learning objective levels 1 (knowledge) and 2 (understanding) $(p=0,01 ; r=-0,20$ and, $p=0,02$; $r=-0,17$, respectively). Hence, no general recommendation favouring either the classical setting or blending learning can be drawn regarding the cognitive domain. However, students' self-indications on the affective domain give preference to blended learning, particularly if inter-professional teamwork is a course objective.
\end{abstract}

\begin{abstract}
Blended Learning bezeichnet eine Kombination von Face-to-Face-Lehre und E-Learning in Bezug auf Wissensvermittlung, Lernaktivitäten der Studierenden und reduzierter Anwesenheit vor Ort. Die vorliegende Kohorten-Studie untersuchte Langzeiteffekte von Blended Learning auf kognitiver Ebene und unmittelbare Einschätzungen des Lerneffekts auf affektiver Ebene im interprofessionellen Berufsfeld „Arbeitsmedizin und Prävention“. Studierende des Bachelorstudiums Physiotherapie der FH Campus Wien absolvierten die Lehrveranstaltung „Arbeitsmedizin und Prävention“ in einem traditionellen Lehr-Lernsetting mit ausschließlicher Face-to-Face-Lehre (Kontrollgruppe, $n=94$ ) oder im Rahmen eines Blended-Learning-Modells (Interventionsgruppe, $\mathrm{n}=93$ ). Verglichen wurde der Langzeiteffekt der kognitiven Lernergebnisse (nach 1,5 Jahren) differenziert nach vier Lernzielstufen nach Bloom. Außerdem wurde evaluiert, wie Studierende den Nutzen des Blended Learnings bezogen auf vier Lernzielstufen nach Krathwohl zur affektiven Leistungsdimension auf einer 6-teiligen Likert Skala einschätzten $(n=282)$. Hinsichtlich der kognitiven Ergebnisse ergaben sich signifikante Unterschiede zugunsten beider Gruppen, wobei die Effektstärken schwach bis mittel ausgeprägt waren. In der höchsten angestrebten kognitiven Lernzielstufe (Analyse) zeigte das traditionelle Lehr-Lernsetting signifikant bessere Ergebnisse im Langzeiteffekt $(p<0,01 ; r=-0,33)$. Die Interventionsgruppe erzielte hingegen signifikant bessere Langzeitergebnisse in den Lernzielstufen 1 (Wissenskenntnisse) und 2 (Verständnis) ( $p=0,01 ; r=-0,20$ bzw. $p=0,02 ; r=-0,17$ ). Hinsichtlich der kognitiven Leistungsdimension kann somit keine allgemeine Empfehlung zugunsten der klassischen Methode oder dem Blended Learning abgeleitet werden. Bezogen auf die affektive Leistungsdimension sprechen Selbstangaben der Studierenden für Blended Learning, vor allem wenn interprofessionelle Teamarbeit Ziel der Lehrveranstaltung ist.
\end{abstract}

\section{Keywords}

Blended learning - learning outcome - e-learning - occupational medicine - prevention - affective domain - cognitive domain

\section{Keywords}

Blended Learning - Lernergebnisse - E-Learning - Arbeitsmedizin - Prävention - Affektive Leistungsdimension - Kognitive Leistungsdimension 


\section{EINLEITUNG}

E-Learning hat im Rahmen der medizinischen Ausbildung seit vielen Jahren einen großen Stellenwert (Ellaway \& Masters, 2008a) und ist als Methode aus der medizinischen Ausbildung nicht mehr wegzudenken (Ellaway \& Masters, 2008b; Ruiz, Mintzer \& Leipzig, 2006).

Im weitesten Sinne ist E-Learning als die Nutzung des Internets innerhalb der Lehre definiert (Ellaway \& Masters 2008a). Im engeren Sinn ist E-Learning ein pädagogischer Ansatz, welcher das Ziel verfolgt, die Lehre räumlich und zeitlich flexibel und lernendenzentriert zu gestalten, um die aktive Zusammenarbeit und Kommunikation zu fördern, sowie die Interaktion (LehrpersonalLehrpersonal, Lehrpersonal-Studierende, StudierendeStudierende) $\mathrm{zu}$ begünstigen (Ellaway \& Masters 2008a). E-Learning wird entweder als reiner OnlineKurs mit keinem bzw. minimalem virtuellen Kontakt zum Lehrpersonal definiert oder auch als Mischung von Online- und Face-to-Face-Aktivitäten verstanden (Ellaway \& Masters, 2008a). Diese Mischung wird als „Blended Learning“ bezeichnet, wo die Vorteile der Faceto-Face-Lehre mit denen des E-Learnings in Bezug auf Wissensvermittlung, Lernaktivitäten der Studierenden und reduzierter Anwesenheit vor Ort kombiniert werden (Babb, Stewart \& Johnson, 2010; Graham, 2006; Kaur, 2013; Koraneekij \& Khlaisang, 2015). Dies entspricht auch dem Begriffsverständnis der hier vorliegenden Kohorten-Studie.

Somit eignet sich das Blended Learning als didaktischer Rahmen für ein Lernsetting, das die Anforderungen von praxisnahen Lernszenarien erfüllt und durch die virtuelle Lernumgebung die Zusammenarbeit zwischen den Studierenden initiiert, ihre Motivation zur Autonomie stärkt und zur Reflexion anregt (Ellaway \& Masters, 2008a; Graham, 2006; Kerres \& de Witt, 2004; Mayer \& Kriz, 2010). Durch diese Art des selbstgesteuerten Lernens wird es Lernenden möglich, den Lernprozess auf die Informationen $\mathrm{zu}$ konzentrieren, die in der individuellen Lernbiografie noch fehlen (Morton et al., 2016). Beim „Vermischen“ (Blending) sind in der Literatur vier verschiedene Modelle benannt, die sich in Bezug auf die zeitliche Abfolge und die dadurch notwendigen Aktivitäten von Studierenden bzw. Lehrpersonal unterscheiden (Graham, 2006). Eines der am häufigsten vorkommenden Blended-LearningModelle ist das Course-Level Blending (Graham, 2006). Bei diesem Modell sind die Face-to-Face- und Online-Phasen chronologisch aufeinanderfolgend, ohne sich zu überschneiden (Graham, 2006). Durch die Online-Phasen im Blended Learning wird das Lernen in die Arbeits- und Lebenswelt der Lernenden hinausgetragen, wodurch der Transfer des Gelernten von der Bildungseinrichtung in die Arbeitswelt unter Umständen leichter gelingt (Ebner, Schön \& Nagler, 2013). Somit stellt das Blended Learning eine geeignete Möglichkeit dar, auf Lehrveranstaltungsebene die berufliche Handlungskompetenz im interprofessionellen Berufsfeld eines Fachbereiches zu erwerben, wie das z.B. im physiotherapeutischen Arbeitsfeld der Arbeitsmedizin und Prävention der Fall ist. Dies ist vor allem dann wichtig, wenn - wie im untersuchten Fachbereich - nicht alle Studierenden im Rahmen eines Praktikums bereits während der Ausbildung ihre Handlungskompetenz erproben können. Das Blended Learning wird methodisch-didaktisch so gestaltet, dass sich mittels Fallbeispielen und durch die vermischte Gestaltung des Lernsettings aufeinanderfolgende Faceto-Face- und Online-Phasen sinnvoll ergänzen (Ellaway \& Masters, 2008a). Der Konstruktion der Fallbeispiele kommt dabei eine große Bedeutung zu. Diese müssen konkret und praxisnah konzipiert sein, sodass diese nicht nur auf die Anwendung von Wissen, sondern auch auf die Umsetzung von Kenntnissen innerhalb dieser praxisrelevanten Problemstellungen fokussieren. Durch dieses Vorgehen wird es möglich, auch Lernergebnisse, die den höheren Lernzielstufen nach Bloom bzw. Krathwohl zugeordnet sind (Anderson \& Krathwohl, CEDEFOP, 2009; Krathwohl, Bloom \& Bertram, 1973), zu erreichen. In Studien zeigt sich, dass gut strukturiertes Blended Learning von Bachelorstudierenden bevorzugt und als mögliche methodische Variante zur Wissensaneignung im Vergleich zu Face-to-Face- bzw. E-Learning allein akzeptiert wird (Gormley, Collins, Boohan, Bickle, \& Stevenson, 2009; Kröncke, 2010; Morton et al., 2016; Ruiz et al., 2006). Dies zeigt sich darin, dass Studierende die Lernumgebung bei Blended Learning als positiv und effektiv wahrnehmen und damit als willkommene Lernumgebung annehmen, welche es schafft, das Interesse an den Inhalten, die (soziale) Interaktion unter den Studierenden, die Lernfreude und die eigene Leistung zu steigern (Garnham \& Kaleta, 2002; Güzer \& Caner 2014; Makhdoom, Khoshhal, Algaidi, Heissam \& Zolaly, 2012). Mittelmäßige bis leistungsstärkere Studierende profitieren mehr von Blended Learning als leistungsschwächere Studierende (Boye, Moen \& Vik, 2012; Owston, York \& Murtha, 2013).

Bezüglich der kognitiven Leistungsdimension zeigen bisherige Studienergebnisse zur Evaluierung von Blended Learning divergierende Ergebnisse (Nordin \& Alias, 2013). In Bezug auf die Wissensspeicherung wird Blended Learning einerseits als minimal effektiver als Face-to-Face-Lehre allein bzw. E-Learning allein beschrieben (Bruff, Fisher, McEwen \& Smith, 2013; Dziuban, Hartmann \& Moskal, 2004; Kröncke, 2010; Means, Toyama, Murphy, Bakia, \& Jones, 2010; Morton et al., 2016). Andererseits gibt es aber auch Evidenz, 
die zeigt, dass Blended Learning nicht effektiver ist als ein traditionelles Lehr-Lernsetting (Güzer \& Caner, 2014; Ilic, Nordin, Glasziou, Tilson \& Villanueva, 2015; Reasons, Valadares \& Slavkin, 2005; Tosun 2015). Das in vielen Studien beschriebene traditionelle LehrLernsetting wird in diesem Artikel als Face-to-FaceLehre (bzw. Kontrollgruppe) bezeichnet. Im Bereich der medizinischen Ausbildung deuten die Ergebnisse darauf hin, dass sich durch den Einsatz von Blended Learning sowohl die klinischen Fähigkeiten inkl. Reflexionsfähigkeit und Clinical Reasoning als auch die Problemlösefähigkeiten und Fähigkeiten des kreativen Denkens verbessern können (Koraneekij \& Khlaisang, 2015; Kröncke, 2010; Rowe, Frantz \& Bozalek, 2012). Bezogen auf die kognitiven Langzeiteffekte von Blended Learning gibt es nur wenige Studien und diese haben ebenso divergierende Aussagen. Peroz, Beuche \& Persoz (2009) zeigen, dass die Wissensspeicherung durch Blended Learning genauso langzeiteffektiv ist wie Face-to-Face-Lehre. Dem gegenüber steht die Aussage von Botezatu, Hult, Tessma, \& Fours (2010), dass fünf Monate nach der Lehrveranstaltung Blended Learning der Face-to-Face-Lehre überlegen ist.

Studienergebnisse zur Auswirkung von Blended Learning auf die affektive Leistungsdimension sind im Vergleich zur Auswirkung auf die kognitive Leistungsdimension eindeutiger: Blended Learning fördert die Entwicklung einer professionellen Haltung und Einstellung bzw. steigert die Nutzung der gelernten Inhalte in der klinischen Praxis (Ilic et al., 2015). Die Steigerung der Motivation und der Zufriedenheit der Studierenden sind weitere positive Effekte (Güzer \& Caner, 2014; Poonam \& Prajana, 2016; River, Currie, Crawford, Betihavas, \& Randall, 2016, Ruiz et al., 2006). Ebenso kann durch Blended Learning der Lerndruck und Abgabestress bei Studierenden und Lehrenden vermindert werden (Nordin \& Alias, 2013). Blended Learning führt vom passiven hin zum aktiven Lernen der Studierenden, weil sie durch reale Fallbearbeitung gezwungen sind, selbst $\mathrm{zu}$ lesen, zu sprechen, zuzuhören und $\mathrm{zu}$ denken und sich selbst zu organisieren (Kaur, 2013). Nicht zuletzt werden durch Blended Learning verschiedene Lerntypen und Lerngeschwindigkeiten berücksichtigt, es kann bedürfnisorientiert gelernt werden und dies fördert einen individuellen und damit personalisierten Lernprozess (Ebner et al., 2013; Ilic et al., 2015).

In bisherigen Studien zu Blended Learning werden die kognitive und die affektive Leistungsdimension wenig detailliert betrachtet. Folgt man den Ausführungen von Bloom (1972) und Krathwohl, Bloom und Bertram (1973), können die beiden Leistungsdimensionen in Lernzielstufen differenziert werden. Die Klassifizierung der einzelnen Lernziele innerhalb einer Leistungsdimension erfolgt dabei von einfachen $\mathrm{zu}$ immer komplexer werdenden Verhaltensweisen. Dabei bauen die Lernziele einer höheren Stufe immer auf die der vorhergehenden Stufe auf (Krathwohl, 2002). Innerhalb der kognitiven Leistungsdimension werden sechs Lernzielstufen definiert: Wissen/Kenntnisse (Lernzielstufe 1), Verständnis (Lernzielstufe 2), Anwendung (Lernzielstufe 3), Analyse (Lernzielstufe 4), Synthese (Lernzielstufe 5) und Beurteilung (Lernzielstufe 6) (CEDEFOP, 2009). Diese Stufen beschreiben die Steigerung der kognitiven Hirnleistung von einfachen, aber konkreten Verhaltensformen zu komplexen und abstrakten (Krathwohl, 2002). Auch die Klassifizierung der affektiven Leistungsdimension, welche im Anschluss an die Klassifizierung im kognitiven Bereich entwickelt wurde, ist durch eine hierarchische Struktur charakterisiert, welche den Prozess der Übernahme von Normen und Werten in die Motiv- und Handlungsstruktur eines Individuums beschreibt. Hier werden fünf Lernzielstufen beschrieben: Wertbeachtung (Lernzielstufe 1), Wertbeantwortung (Lernzielstufe 2), Wertung (Lernzielstufe 3), Wertordnung (Lernzielstufe 4) und Wertverinnerlichung (Lernzielstufe 5) (CEDEFOP, 2009). Je höher die in der affektiven Leistungsdimension erreichte Lernzielstufe eines Individuums ist, desto mehr kann von einer ,entwickelten Persönlichkeit“" gesprochen werden.

Zusammenfassend kann festgestellt werden, dass bisherige Studienergebnisse die Lernergebnisse von Blended Learning innerhalb der kognitiven und affektiven LeistungsdimensionnichtdifferenziertnachLernzielstufen betrachten. Außerdem ist der Langzeiteffekt von Blended Learning versus Face-to-Face-Lehre nicht gesichert. Das Ziel dieser Studie ist es daher, herauszufinden, wie sich durch Blended Learning (im Vergleich zur Face-to-FaceLehre) die kognitive Leistungsdimension, differenziert nach Lernzielstufen, langfristig entwickelt.

Ebenso steht im Fokus, ob durch Blended Learning ein Einfluss auf die verschiedenen Lernzielstufen der affektiven Leistungsdimension unmittelbar nach der Lehrveranstaltung feststellbar ist.

\section{Fragestellung}

Folgende zwei Fragestellungen wurden im Rahmen dieser Kohorten-Studie untersucht:

1. Gibt es einen Unterschied bezogen auf den Langzeiteffekt der Lernergebnisse der kognitiven Leistungsdimension (differenziert nach Lernzielstufen) zwischen der Face-to-Face-Lehre und Blended Learning?

2. Wie schätzen Studierende Blended Learning zur Entwicklung der affektiven Leistungsdimension (differenziert nach Lernzielstufen) ein? 


\section{METHODE}

\section{Intervention}

In drei aufeinanderfolgenden Jahrgängen (Kohorten 2-4) wurden 18 Einheiten (à 45 Minuten) der Lehrveranstaltung „Arbeitsmedizin und Prävention“ (im Studiengang Physiotherapie, FH Campus Wien) als Blended Learning abgehalten. Das Blended Learning entsprach dem Modell des Course-Level Blending mit drei aufeinanderfolgenden Phasen. Die erste Phase wurde als Face-to-FaceLehre abgehalten und beinhaltete eine thematische Einführung in das interprofessionelle Arbeitsfeld der Arbeitsmedizin und Prävention sowie Erläuterungen zum Ablauf der anschließenden Online-Phase. In dieser Phase erstellten die Studierenden in Kleingruppen (3-4 Studierende) als Erstes ein Angebot arbeitsmedizinischer physiotherapeutischerBeratung und Intervention zu jeweils einer Projektausschreibung eines realen Unternehmens und befanden sich damit in einer berufsspezifischen Rolle. In einem zweiten Schritt wechselten die Studierenden die Rolle und bewerteten als Unternehmen ein von einer anderen Kleingruppe erstelltes Angebot. In beiden Rollen bereiteten sie sich auf ein Rollenspiel in der nachfolgenden Lehrveranstaltungssequenz, die wieder als Face-to-FaceLehre gestaltet war, vor.

In der Kontrollgruppe fand die Lehrveranstaltung als ausschließliche Face-to-Face-Lehre statt. Das Rollenspiel wurde in verkürzter Form, unter Anleitung der Lehrveranstaltungsleitung und ohne vorherige Auseinandersetzung mit dem Thema durchgeführt.

Der Langzeiteffekt wird 1,5 Jahre nach Abschluss jener Lehrveranstaltung gemessen, die die untersuchte kognitive Leistungsdimension in ihren Lernergebnissen ausweist. Zu diesem Zeitpunkt stehen die Studierenden wenige Wochen vor dem Abschluss ihrer Ausbildung und damit vor der Erlangung ihrer Berufsbefähigung zur Ausübung der Physiotherapie. Das Kompetenzprofil der Physiotherapeutinnen und Physiotherapeuten Österreichs setzt sich aus mehreren Bausteinen zusammen, die als QualifikationszieledurchdieLehrveranstaltungendes 1.bis 6. Semesters des Bachelorstudiums curricular angesteuert werden (Physio Austria, 2016). Lernergebnisse der kognitiven Leistungsdimension aus der Lehrveranstaltung „Arbeitsmedizin und Prävention“ werden im Verlauf des weiteren Studiums in Lehrveranstaltungen aufgegriffen, die diese Lernergebnisse als curricular definierte Lernvoraussetzungen aufweisen. Damit ist gewährleistet, dass sich die Studierenden immer wieder mit den Inhalten auseinandersetzen. Auf welcher Lernzielstufe die Lernergebnisse der kognitiven Leistungsdimension am Ende der Ausbildung abrufbar sind und damit als Baustein des Kompetenzprofils zur Verfügung stehen, steht im Fokus dieser Untersuchung.

\section{Teilnehmende}

Zur Beantwortung der ersten Fragestellung wurden alle Studierenden der Kontroll-Kohorte 1 und BlendedLearning-Kohorte 2 eingeschlossen, die sich 1,5 Jahre nach Abschluss der Lehrveranstaltung „Arbeitsmedizin und Prävention" noch in der Ausbildung befanden und das Studium bis dahin in der Regelstudiendauer absolviert hatten. Die Zuweisung der Teilnehmenden in die BlendedLearning- bzw. Kontroll-Kohorte erfolgte durch deren Zugehörigkeit zu einem Ausbildungsjahrgang, diese Zuweisung war nicht randomisiert und/oder verdeckt. Alle Studierende der Kontroll-Kohorte 1 wurden in der Lehrveranstaltung „Arbeitsmedizin und Prävention“ ausschließlich durch Face-to-Face-Lehre unterrichtet. Im darauffolgenden Studienjahr wurden alle Studierenden der Blended-Learning-Kohorte 2 in der Lehrveranstaltung „Arbeitsmedizin und Prävention“ ausschließlich mit Blended Learning unterrichtet.

Für die zweite Fragestellung wurden Studierende der Kohorten 2 bis 4, die an der Blended-LearningLehrveranstaltung teilgenommen hatten, unmittelbar nach der Lehrveranstaltung mit einem Einschätzungsbogen zur affektiven Leistungsdimension befragt.

\section{Outcome Assessments}

Blended-Learning- und Kontroll-Kohorte wurden 1,5 Jahre nach Lehrveranstaltungsende anhand eines schriftlichen Tests befragt. Mithilfe von fünf MultipleChoice- und einer Single-Choice-Testaufgabe wurden die für die Lehrveranstaltung definierten Lernergebnisse innerhalb der kognitiven Leistungsdimension abgefragt. Die sechs Testaufgaben waren den ersten vier der sechs Lernzielstufen nach Bloom (Bloom, 1972) zugeordnet. Die Lernergebnisse der Lernzielstufe 1 (Wissen/Kenntnisse) und 2 (Verständnis) wurden nach monoprofessionellen (a) bzw. interprofessionellen (b) Aspekten differenziert betrachtet. So wurden Wissen/Kenntnisse einerseits monoprofessionell, auf den arbeitsmedizinischen Kontext bezogen, abgefragt (Lernzielstufe 1a und 2a). Andererseits war Grundlagenwissen und -verständnis, das die Basis für ein Rollenverständnis und ein teamorientiertes interprofessionelles Handeln bildet, Inhalt von weiteren Testaufgaben (Lernzielstufe $1 \mathrm{~b}$ und 2b; siehe Tabelle 1). Aufbauend auf den Lernergebnissen der unteren Lernzielstufen, stehen Lernergebnisse, die die Fähigkeit erfordern, Wissen anzuwenden (Lernzielstufe 3) sowie die Fähigkeit zur Analyse von Sachverhalten (Lernzielstufe 4).

Zur Überprüfung des generellen Leistungsniveaus der StudierendenderKontroll-undBlended-Learning-Kohorte wurden die Abschlussnoten aller Lehrveranstaltungen des entsprechenden Beobachtungssemesters verglichen. 
Tabelle 1: Lernziele der Lehrveranstaltung „Arbeitsmedizin und Prävention“ und deren Zuordnung zu den Lernzielstufen der kognitiven Leistungsdimension in Anlehnung an Bloom (Bloom, 1972)

\begin{tabular}{|c|c|c|c|c|c|}
\hline \multicolumn{2}{|c|}{ Lernzielstufe } & \multirow[b]{2}{*}{$\begin{array}{l}\text { Lernziel } \\
\\
\text { Erkennen der } \\
\text { richtigen Definition zu } \\
\text { tätigkeitsassoziierten } \\
\text { Erkrankungen }\end{array}$} & \multirow[b]{2}{*}{$\begin{array}{c}\text { Testaufgabe } \\
\\
\text { Bitte kreuzen Sie jene } \\
\text { Definition an, die Ihrer } \\
\text { Meinung nach auf } \\
\text { Tätigkeitsassoziierte } \\
\text { Erkrankungen zutrifft }\end{array}$} & \multicolumn{2}{|c|}{ Lösung } \\
\hline \multirow{2}{*}{ 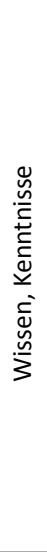 } & $1 a$ & & & $\begin{array}{r}\text { Tätigkeitsassoziierte Erkrankun } \\
\square \text { bei denen die Arbeit ein signif } \\
\bigotimes \text { bei denen die Arbeit der eir } \\
\text { 冈 die wissenschaftlich nachs } \\
\text { Einwirkungen eines bestimm } \\
\text { Arbeitsplatz ent } \\
\text { ख die bei Personengruppen durch } \\
\text { der Arbeit signifikant }\end{array}$ & $\begin{array}{l}\text { gen sind Beschwerdebilder, } \\
\text { fikanter Entstehungsfaktor ist. } \\
\text { zzige Entstehungsfaktor ist. } \\
\text { gewiesen eindeutig durch } \\
\text { ten Belastungsfaktors am } \\
\text { standen sind. } \\
\text { bestimmte Belastungsfaktoren } \\
\text { häufiger auftreten. }\end{array}$ \\
\hline & $1 b$ & $\begin{array}{l}\text { Identifizieren von } \\
\text { Kernaufgaben der } \\
\text { Physiotherapie im } \\
\text { interprofessionellen } \\
\text { Berufsfeld der } \\
\text { Arbeitsmedizin }\end{array}$ & $\begin{array}{l}\text { Bitte kreuzen Sie jene Items } \\
\text { an, die Ihrer Meinung } \\
\text { nach zu den Kernaufgaben } \\
\text { einer Physiotherapeutin/ } \\
\text { eines Physiotherapeuten im } \\
\text { Betrieb/Unternehmen zählen }\end{array}$ & $\begin{array}{c}\square \text { Identifizierung von } \\
\text { Belastungsfaktoren } \\
\square \text { Motivation zur } \\
\text { Auseinandersetzung mit der } \\
\text { Gesundheit } \\
\square \text { Arbeitsplatzanalysen } \\
\square \text { Arbeitsplatzgestaltung } \\
\square \text { Anpassung der Arbeit an den } \\
\text { Menschen } \\
\end{array}$ & $\begin{array}{c}\text { 冈 Unfallverhütung } \\
\text { Vorsorgeuntersuchungen } \\
\text { 冈 Aufstellung von } \\
\text { Grenzwerten } \\
\text { Beschäftigung der } \\
\text { Mitarbeitenden entsprechend } \\
\text { ihrer individuellen Eignung } \\
\text { 冈 Behandlung von Patienten/- } \\
\text { innen mit Berufskrankheiten }\end{array}$ \\
\hline \multirow[b]{2}{*}{ 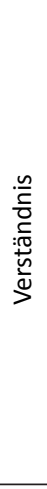 } & $2 a$ & $\begin{array}{l}\text { Verstehen von } \\
\text { Belastungsfaktoren } \\
\text { als Resultat der } \\
\text { Arbeitsumgebung }\end{array}$ & $\begin{array}{c}\text { Bitte kreuzen Sie jene } \\
\text { Belastungen am Arbeitsplatz } \\
\text { an, die Ihrer Meinung nach } \\
\text { aus der Arbeitsumgebung } \\
\text { resultieren }\end{array}$ & $\begin{array}{c}\square \text { Schadstoffe } \\
\square \text { Lärm } \\
\square \text { Vibration } \\
\square \text { Beleuchtung }\end{array}$ & $\begin{array}{c}\text { 冈 Arbeitszeit } \\
\text { 冈 Informationsaufnahme und } \\
\text {-verarbeitung } \\
\text { 冈 Monotonie } \\
\text { 冈 Zeitdruck }\end{array}$ \\
\hline & $2 b$ & $\begin{array}{l}\text { Aufgabenfelder der } \\
\text { Verhältnisergonomie } \\
\text { aus dem } \\
\text { Begriffsverständnis } \\
\text { in einem } \\
\text { interprofessionellen } \\
\text { Kontext ableiten }\end{array}$ & $\begin{array}{c}\text { Bitte kreuzen Sie jene Items } \\
\text { an, die Ihrer Meinung nach } \\
\text { beschreiben, womit sich } \\
\text { die Verhältnisergonomie } \\
\text { beschäftigt }\end{array}$ & $\begin{array}{l}\text { Verhältnisergonomie beschäftigt } \\
\text { sich mit } \\
\square \text { der Interaktion von Mensch } \\
\text { und technischen Systemen. } \\
\square \text { der Gestaltung der } \\
\text { Arbeitsumwelt. } \\
\square \text { der Gestaltung der } \\
\text { Freizeitumwelt. } \\
\square \text { der Entwicklung von Regeln } \\
\text { zur Arbeitsplatzgestaltung. }\end{array}$ & $\begin{array}{c}\otimes \text { der Förderung } \\
\text { individuellen, } \\
\text { gesundheitsgerechten } \\
\text { Verhaltens. } \\
\text { der Gestaltung der } \\
\text { Arbeitsaufgabe. } \\
\text { der Anpassung der } \\
\text { Arbeitsorganisation. }\end{array}$ \\
\hline 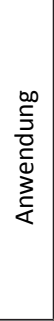 & 3 & $\begin{array}{l}\text { Belastungs- und } \\
\text { Beanspruchungsmodell } \\
\text { auf Aussagen zur } \\
\text { Relation zwischen } \\
\text { Belastung und } \\
\text { Beanspruchung } \\
\text { anwenden }\end{array}$ & $\begin{array}{l}\text { Bitte kreuzen Sie jene } \\
\text { Aussagen an, die Ihrer } \\
\text { Meinung nach auf das } \\
\text { Belastungs-Beanspruchungs- } \\
\text { Modell zutreffen }\end{array}$ & $\begin{array}{c}\square \text { Belastung ist abhängig von } \\
\text { Belastungshöhe und Dauer. } \\
\square \text { Die Dauerleistungsgrenze ist } \\
\text { individuell. } \\
\square \text { Die Beanspruchung ist } \\
\text { individuell. } \\
\square \text { Individuelle Ressourcen } \\
\text { können die Beanspruchung } \\
\text { reduzieren. }\end{array}$ & 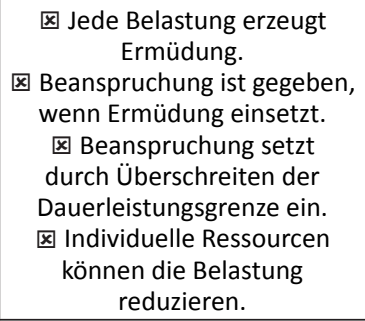 \\
\hline $\begin{array}{l}\frac{\tilde{N}}{\frac{0}{\pi}} \\
\frac{\tilde{c}}{<}\end{array}$ & 4 & $\begin{array}{c}\text { Strukturieren } \\
\text { von Elementen } \\
\text { eines Angebots } \\
\text { auf der Basis des } \\
\text { Physiotherapeutischen } \\
\text { Prozesses } \\
\text { Erkennen von } \\
\text { Zusammenhängen, } \\
\text { Differenzieren nach } \\
\text { Reihenfolge }\end{array}$ & $\begin{array}{l}\text { Ein Unternehmen schreibt } \\
\text { ein Projekt zur betrieblichen } \\
\text { Gesundheitsförderung aus. } \\
\text { Sie als PT bewerben sich } \\
\text { und legen nach erfolgter } \\
\text { Arbeitsplatzbegehung ein } \\
\text { Angebot vor. Bringen Sie die } \\
\text { Elemente des Angebots auf } \\
\text { der Basis des PT-Prozesses in } \\
\text { eine sinnvolle Reihenfolge }\end{array}$ & $\begin{array}{c}\text { Vorgegebene Items in richtiger } \\
\text { Reihenfolge: } \\
\text { 1. Tätigkeitsanalyse } \\
\text { 2. Bewertung der Arbeitsleistung } \\
\text { 3. Identifizierte } \\
\text { Belastungsfaktoren } \\
\text { 4. Beitragende Faktoren } \\
\text { 5. Zieldefinition der PT- } \\
\text { Intervention }\end{array}$ & $\begin{array}{l}\text { 6. oder 7. Maßnahmen } \\
\text { zur Optimierung der } \\
\text { Pausengestaltung } \\
\text { 6. oder 7. Maßnahmen } \\
\text { der Verhältnis- u./o. } \\
\text { Verhaltensergonomie } \\
\text { 8. Setting der PT-Intervention } \\
\text { 9. Materialien für die PT- } \\
\text { Intervention } \\
\text { 10. Kostendarstellung }\end{array}$ \\
\hline
\end{tabular}

$a=$ monodisziplinäre Aspekte stehen im Vordergrund; $b=$ interdisziplinäre Aspekte stehen im Vordergrund; $\square$ richtige Antwort; $囚$ falsche Antwort

DreiJahrgänge(Kohorten2-4), dieanderLehrveranstaltung mit Blended Learning teilgenommen hatten, füllten unmittelbar nach Abschluss der Lehrveranstaltung einen Einschätzungsbogen aus. Dieser Einschätzungsbogen (bestehend aus sechs Fragen, siehe dazu Tabelle 4) ermittelte, inwieweit die Studierenden Blended Learning für die Entwicklung der affektiven Leistungsdimension nutzen konnten. Die abgefragten Lernergebnisse reichten von den Lernzielstufen 1 (Wertbeachtung) und 2 (Wertbeantwortung) in der Form des Bewusstseins und Reagierens bis zu den Lernzielstufen 3 (Wertung) und 4 (Wertordnung) nach Krathwohl (CEDEFOP, 2009), die auf das Bewerten und den Aufbau eines Wertesystems abzielten. Für die Einschätzung diente bei fünf Fragen 


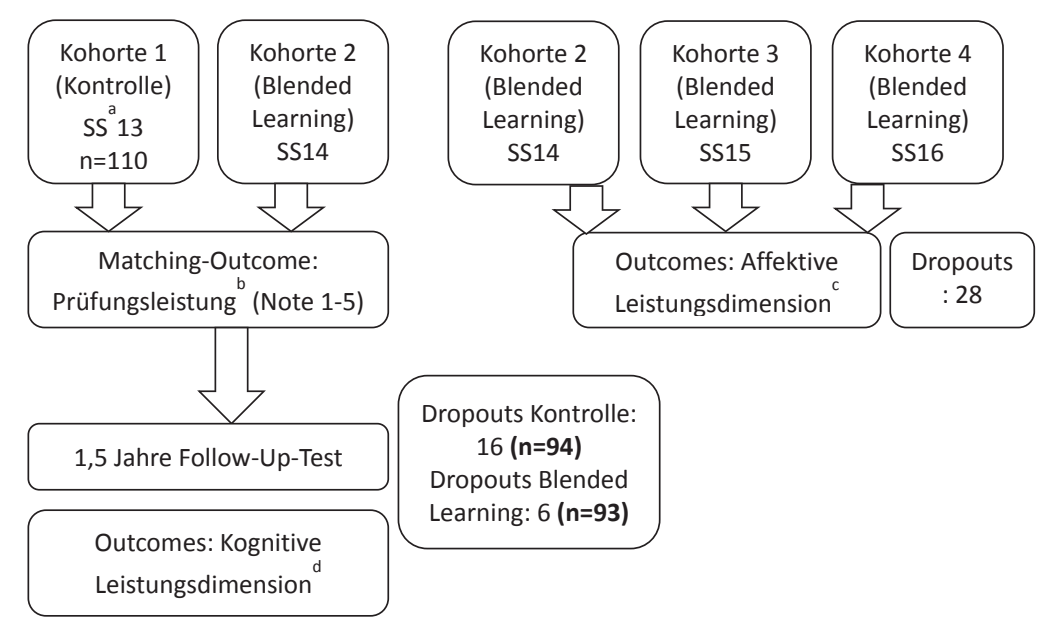

Abbildung 1: Studiendesign und Teilnehmenden-Flussdiagramm

${ }^{a}$ SS=Sommersemester, in dem die Lehrveranstaltung stattgefunden hat

${ }^{b}$ Durchschnittliches Prüfungsergebnis aller im Beobachtungssemester abgeschlossenen Lehrveranstaltungen

' Einschätzungen auf einer Likert-Skala zu vier Lernzielstufen der affektiven Leistungsdimension nach Krathwohl (CEDEFOP, 2009)

${ }^{d}$ Sechs Fragen zu vier Lernzielstufen der kognitiven Leistungsdimension in Anlehnung an Bloom (Bloom, 1972)

eine 6-teilige und bei einer Frage (Lernzielstufe 2) eine 3-teilige Likert Skala. Ein Überblick über Studiendesign und Teilnehmenden-Flussdiagramm sind in Abbildung 1 zusammengestellt.

\section{Statistische Analysen}

Der Test für die Beantwortung der ersten Fragestellung beinhaltete sechs Testaufgaben. Fünf der sechs Testaufgaben enthielten eine variierende Anzahl an Antwortmöglichkeiten. Dabei wurde der Prozentsatz der richtig angekreuzten bzw. richtig nicht angekreuzten Antworten berechnet; $100 \%$ bedeutet, die Testaufgabe wurde komplett richtig beantwortet. Die Single-ChoiceTestaufgabe (Lernzielstufe 1a) konnte entweder richtig oder falsch beantwortet werden.

Die Normalverteilung der Daten (Testaufgabe der Lernzielstufen 1b und 2-4) wurde mittels ShapiroWilk-Test und zusätzlicher grafischer Kontrolle von Histogrammen mit Normalverteilungskurven überprüft. Da bei keinem der Outcome-Parameter ein metrisches Skalierungsniveau und eine Normalverteilung gegeben war, kamen nicht-parametrische Darstellungs- und Prüfverfahren zur Anwendung. Zur Prüfung von Unterschieden zwischen Blended-Learning- und KontrollKohorte wurde der Mann-Whitney-U-Test angewendet. Der Unterschied zwischen den beiden Gruppen in der Lernzielstufe 1a (dichotomes Datenniveau) wurde mittels Chi-Quadrat-Test berechnet. Das Signifikanzniveau $(\alpha)$ wurde auf $\mathrm{p}<0,05$ festgesetzt.

Für die Lernzielstufen $1 \mathrm{~b}$ und 2-4 wurde die nichtparametrische Effektgröße r, basierend auf der Z-Statistik, berechnet $(\mathrm{r}=\mathrm{Z} / \sqrt{ } \mathrm{N})$. Dieser Wert ist wie folgt $\mathrm{zu}$ interpretieren: $<0,3$ entspricht einer kleinen
Effektgröße, 0,3 bis $<0,5$ einer mittleren und $\geq 0,5$ einer großen Effektgröße (Field, 2013). In weiterer Folge wurde $r^{2}\left(r^{2}=Z^{2} / N\right)$ kalkuliert und mit 100 multipliziert. Dadurch wird ausgedrückt wie viel Prozent der Varianz der Lernergebnisse auf die Intervention zurückzuführen sind (Ivarsson, Anderson, Johnson, \& Lindwall, 2013; Tomczak \& Tomczak, 2014).

Hinsichtlich der zweiten Fragestellung wurden Einschätzungsbögen zur affektiven Leistungsdimension herangezogen und mit beschreibender Statistik dargestellt (Häufigkeitsverteilungen, Median, 25\%- und 75\%Quartile).

\section{RESULTATE}

Für die Beantwortung der ersten Fragestellung konnten 187 vollständig ausgefüllte schriftliche Tests herangezogen werden (Blended-Learning-Kohorte 2 $\mathrm{n}=93$, Kontroll-Kohorte $1 \mathrm{n}=94) .16$ Studierende der Kontroll-Kohorte 1 und sechs Studierende der BlendedLearning-Kohorte 2 waren am Tag der Datenerhebung nicht anwesend und füllten keinen Test aus. Allgemeine Stichprobencharakteristika sind in Tabelle 2 zusammengefasst.

Die beiden Kohorten unterschieden sich nicht signifikant hinsichtlich der durchschnittlichen Prüfungsleistung im Semester der Lehrveranstaltung ( $U=198,50, z=-0,41$, $\mathrm{p}=0,97, \mathrm{r}=-0,07)$. Im 1,5-jährigem Follow-Up erreichte die Blended-Learning-Kohorte 2 bessere Resultate in den Lernzielstufen $1 b(\mathrm{~b}=3428,50, \mathrm{z}=-2,67, \mathrm{p}=0,01$, $\mathrm{r}=-0,20)$ und $2 \mathrm{a}(\mathrm{U}=3564,50, \mathrm{z}=-2,28, \mathrm{p}=0,02, \mathrm{r}=-0,17)$; $3,8 \%$ bzw. 2,8\% der Varianz können dabei auf die Intervention zurückgeführt werden. In der Lernzielstufe 4 schnitt die Kontroll-Kohorte $1(\mathrm{U}=2704,50, \mathrm{z}=-4,56$, 
Tabelle 2: Allgemeine Charakteristika der vier Stichproben-Kohorten, getrennt nach Assessments zur kognitiven und affektiven Leistungsdimension

\begin{tabular}{|c|c|c|c|c|c|}
\hline & $\begin{array}{c}\text { Kohorte } 1, \text { kognitiv } \\
\text { SS }^{\mathrm{a}} 13 \\
(n=110)\end{array}$ & $\begin{array}{c}\text { Kohorte } 2 \text {, kognitiv } \\
\text { SS } 14 \\
(n=99)\end{array}$ & $\begin{array}{c}\text { Kohorte 2, affektiv } \\
\text { SS } 14 \\
(n=109)\end{array}$ & $\begin{array}{c}\text { Kohorte 3, affektiv } \\
\text { SS } 15 \\
(n=98)\end{array}$ & $\begin{array}{c}\text { Kohorte 4, affektiv } \\
\text { SS } 16 \\
(n=103)\end{array}$ \\
\hline Geschlecht: n (\%) & $\begin{array}{l}M^{d}: 28(25,5 \%) \\
W^{e}: 82(74,5 \%)\end{array}$ & $\begin{array}{l}M: 27(27,3 \%) \\
W: 72(72,7 \%)\end{array}$ & $\begin{array}{l}\text { M: } 32(29,4 \%) \\
\text { W: } 77(70,6 \%)\end{array}$ & $\begin{array}{l}\text { M: } 33(33,7 \%) \\
\text { W: } 65(66,3 \%)\end{array}$ & $\begin{array}{l}M: 33(32,0 \%) \\
W: 70(68,0 \%)\end{array}$ \\
\hline Alterf: MW (SD) & $23,6(4,7)$ & $23,2(4,9)$ & $23,4(5,3)$ & $23,0(4,3)$ & $23,2(3,4)$ \\
\hline $\begin{array}{l}\text { Zulassungsvoraus- } \\
\text { setzung: } \mathrm{n}(\%)\end{array}$ & $\begin{array}{l}\text { AHS: } 72(65,5 \%) \\
\text { BHS }^{\text {h: }} 32(29,1 \%) \\
\text { Sonstige': } 6(5,5 \%)\end{array}$ & $\begin{array}{c}\text { AHS: } 61(61,6 \%) \\
\text { BHS: } 32(32,3 \%) \\
\text { Sonstige: } 6(6,1 \%)\end{array}$ & $\begin{array}{c}\text { AHS: } 66(60,6 \%) \\
\text { BHS: } 32(29,4 \%) \\
\text { Sonstige: } 8(7,3 \%) \\
\text { Nostrij: } 3(2,8 \%)\end{array}$ & $\begin{array}{c}\text { AHS: } 62(63,3 \%) \\
\text { BHS: } 27(27,6 \%) \\
\text { Sonstige: } 9(9,2 \%)\end{array}$ & $\begin{array}{c}\text { AHS: } 60(58,3 \%) \\
\text { BHS: } 31(30,1 \%) \\
\text { Sonstige: } 11(10,7 \%) \\
\text { Nostri :1 }(1,0 \%)\end{array}$ \\
\hline
\end{tabular}

a Stichprobe zum Assessment der kognitiven Leistungsdimension 1,5 Jahre nach Abschluss der Lehrveranstaltung im SS13 (Kohorte 1) bzw. im SS14 (Kohorte 2)

${ }^{b}$ Stichprobe zum Assessment der affektiven Leistungsdimension unmittelbar nach Abschluss der Lehrveranstaltung

'SS = Sommersemester, in dem die Lehrveranstaltung stattgefunden hat

${ }^{d} M=$ männlich

${ }^{e} W=$ weiblich

${ }^{f}$ Alter in Jahren zum Zeitpunkt des Abschlusses der Lehrveranstaltung; $M W=$ Mittelwert; $S D=$ Standardabweichung

${ }^{g}$ AHS = Allgemeinbildende höhere Schule

${ }^{h}$ BHS $=$ Berufsbildende höhere Schule

'Sonstige = Berufsreifeprüfung, Schule für den medizinisch-technischen Fachdienst mit Zusatzprüfungen, anerkannte Studienberechtigungsprüfung, Externisten-Reifeprüfung, Abschlusszeugnis einer facheinschlägigen Berufsbildenden mittleren Schule

${ }^{j}$ Nostri = Studienzulassung über Nostrifizierungsverfahren

Tabelle 3: Ergebnisse des Tests und Zuordnung zu den Lernzielstufen der kognitiven Leistungsdimension in Anlehnung an Bloom (Bloom, 1972)

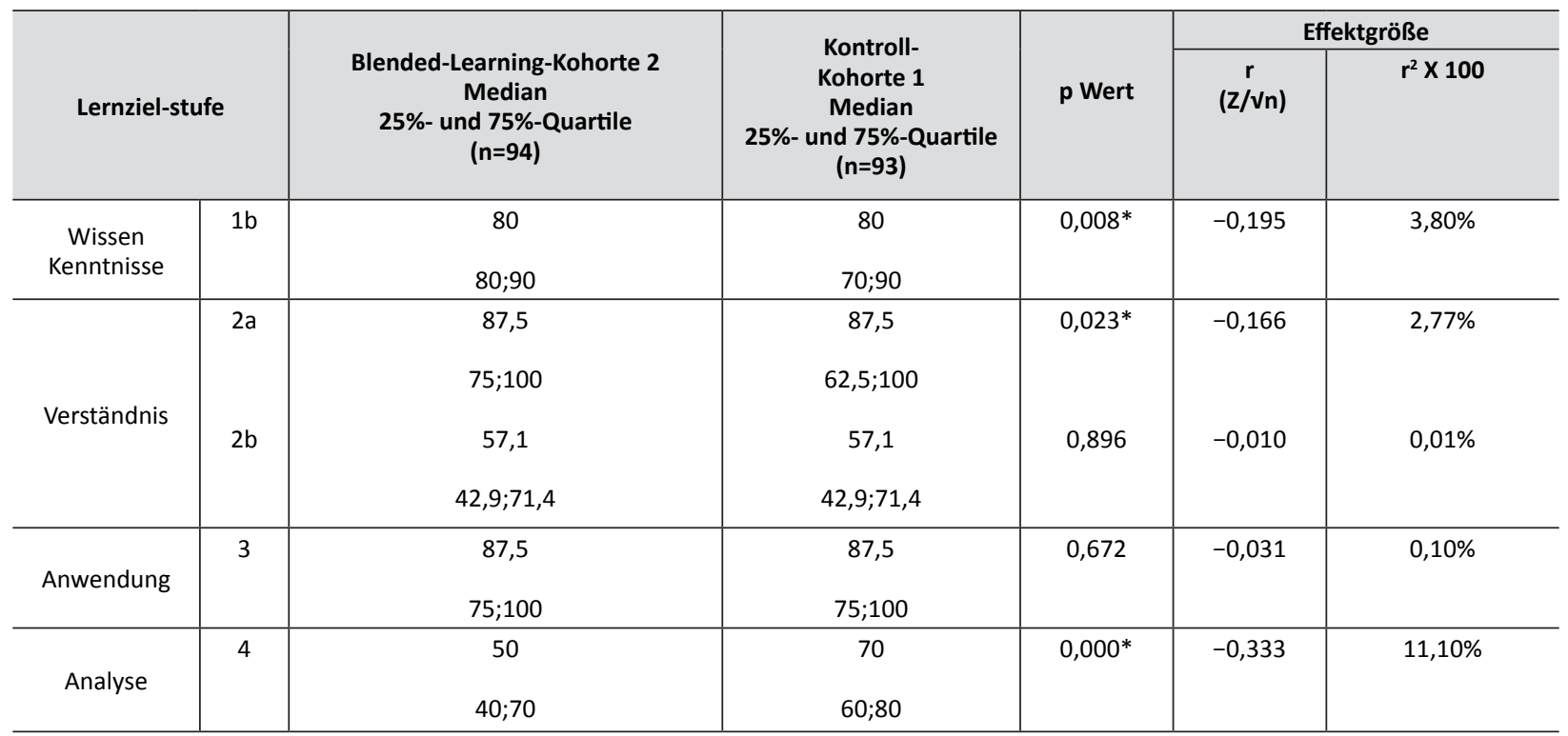

$a=$ monoprofessionelle Aspekte stehen im Vordergrund; $b=$ interprofessionelle Aspekte stehen im Vordergrund

Wertung von 0 bis 100: 0 = keine richtige Antwort; 100 = vollständig richtig beantwortete Testaufgabe

* signifikanter Unterschied zwischen der Blended-Learning- und der Kontroll-Kohorte (Mann-Whitney-U-Test)

Anmerkung: Resultat der Lernzielstufe 1 a mit dichotomer Merkmalsausprägung $\left(X^{2(1, n=187)=2,20, p=0,18)}\right.$

$\mathrm{p}<0,01, \quad \mathrm{r}=-0,33)$ signifikant besser ab; $11,1 \%$ der Varianz konnten dabei auf die Intervention zurückgeführt werden. Abbildung 2 zeigt Ergebnisse der MultipleChoice-Fragen, getrennt nach Intervention und Kontrolle; Details dieser Outcomes sind in Tabelle 3 ersichtlich. Die Testaufgabe zur Lernzielstufe 1a (Single-ChoiceTestaufgabe) wurde in der Blended-Learning-Kohorte 2 von 13 Personen (vs. 21 Personen in der Kontroll-Kohorte 1) richtig und von 80 Personen (vs. 73 Personen in der Kontroll-Kohorte 1) falsch beantwortet. Zwischen den beiden Kohorten bestand kein signifikanter Unterschied $\left(\mathrm{X}^{2}(1, \mathrm{n}=187)=2,20, \mathrm{p}=0,18\right)$.

Hinsichtlich der zweiten Fragestellung füllten 282 Studierende aus drei Jahrgängen, die mit dem 

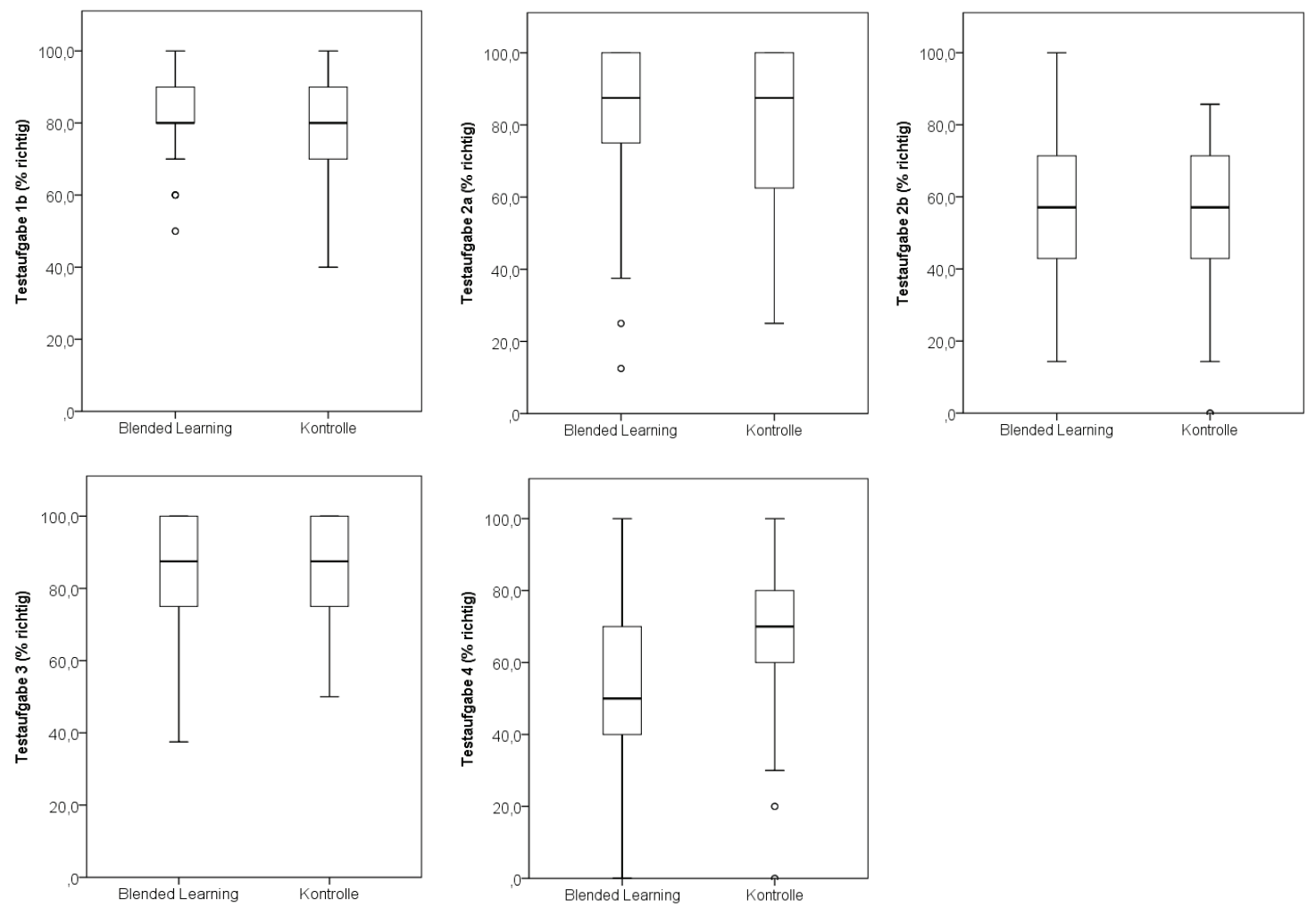

Abbildung 2: Testergebnisse der Blended-Learning-Kohorte 2 (n=93) und Kontroll-Kohorte 1 ( $n=94)$ 1,5 Jahre nach Abschluss der Lehrveranstaltung

$1 b: p=0,01 ; 2 a: p=0,02 ; 2 b: p=0,90 ; 3: p=0,67 ; 4: p<0,01$

Blended-Learning-Modell angeleitet wurden, einen Einschätzungsbogen zur affektiven Leistungsdimension unmittelbar nach der Lehrveranstaltung aus. Im Einschätzungsbogen erfolgte die Zuordnung der Einschätzung durch das Ankreuzen eines Emoticons (siehe Tabelle 4, Spalte 3 bis 5). Bis auf 28 Studierende, die zum Zeitpunkt der Einschätzung entweder nicht anwesend waren oder an der Einschätzung nicht teilnehmen wollten, füllten alle Studierenden der drei Jahrgänge den Einschätzungsbogen aus. In der Lernzielstufe 1 (Wertbeachtung), 3 (Wertung) und 4 (Wertordnung) der affektiven Leistungsdimension wurde der Nutzen von Blended Learning mit einer 6-teiligen Likert Skala anhand von Selbstangaben eingeschätzt. Dabei entspricht 1 der bestmöglichen und 6 der schlechtesten Beurteilung. Die Mediane lagen dabei zwischen 1 und 3. Somit zeigte sich, dass die Studierenden das Blended-LearningModell für die affektive Leistungsdimension als eher fördernd einstuften (siehe Tabelle 4). Die Frage nach der Anforderung der Lehrveranstaltung an die Studierenden (Lernzielstufe 2 Wertbeantwortung) lässt erkennen, dass sich die meisten Studierenden $(257 ; 91 \%)$ genau richtig gefordert fühlten, $16(6 \%)$ Studierende waren überfordert und $9(3 \%)$ Studierende waren unterfordert.

\section{DISKUSSION}

Die vorliegende Kohorten-Studie stellt einen Beitrag zur Evaluierung von Blended Learning innerhalb der medizinischen und gesundheitswissenschaftlichen Ausbildung dar. Die nach Lernzielen differenzierte Betrachtung der kognitiven und affektiven Leistungsdimension gibt erste Hinweise darauf, welchen Einfluss Blended Learning auf die einzelnen Lernzielstufen haben kann.

\section{Diskussion der Ergebnisse der kognitiven Leistungsdimension}

Die differenzierte Betrachtung nach monoprofessionellen (Lernzielstufe 1a und 2a) und interprofessionellen (Lernzielstufe $1 \mathrm{~b}$ und 2b) Aspekten zeigt auf Lernzielstufe 1 auf interprofessioneller Ebene einen signifikanten Unterschied und auf monoprofessioneller Ebene keinen signifikanten Unterschied zwischen den Versuchskohorten. Auf der Lernzielstufe 2 verhält es sich umgekehrt: Monoprofessionell zeigt sich ein signifikanter Unterschied zwischen den Versuchskohorten, interprofessionell jedoch nicht. Diese gegensätzlichen 
Tabelle 4: Ergebnisse des Einschätzungsbogens und Zuordnung zu den Lernzielstufen der affektiven Leistungsdimension nach Krathwohl (CEDEFOP, 2009)

\begin{tabular}{|c|c|c|c|c|c|c|c|c|c|c|}
\hline \multirow{3}{*}{ Lernzielstufe } & \multirow{3}{*}{$\begin{array}{l}\text { Lernziel } \\
\text { der Lehrveranstaltung } \\
\text { „Arbeitsmedizin und } \\
\text { Prävention“ }\end{array}$} & & \multicolumn{6}{|c|}{$\begin{array}{c}\text { Likert Skala } \\
\text { (Häufigkeiten) }\end{array}$} & & \multirow{3}{*}{$\begin{array}{l}\text { Median } \\
25 \%-; \\
75 \%- \\
\text { Quantile }\end{array}$} \\
\hline & & & (ब9) & (30) & (ब) & (9-9) & $\stackrel{\circ 000}{\circ}$ & 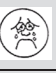 & & \\
\hline & & & 1 & 2 & 3 & 4 & 5 & 6 & & \\
\hline $\begin{array}{c}1 \\
\text { Wertbeachtung }\end{array}$ & $\begin{array}{l}\text { Bewusstsein über } \\
\text { Sachverhalte und } \\
\text { Klarheit über Aufgaben } \\
\text { erlangen }\end{array}$ & $\begin{array}{c}\text { Die Aufgaben } \\
\text { waren klar, ich habe } \\
\text { gewusst, was wann zu } \\
\text { tun ist. } \\
\end{array}$ & 44 & 96 & 92 & 39 & 9 & 2 & $\begin{array}{l}\text { Ich habe mich nicht } \\
\text { ausgekannt, es war } \\
\text { total verwirrend. }\end{array}$ & $\begin{array}{c}3 \\
2 ; 3\end{array}$ \\
\hline \multirow{2}{*}{$\begin{array}{c}3 \\
\text { Wertung }\end{array}$} & $\begin{array}{c}\text { Begründete } \\
\text { Entscheidungsfindung, } \\
\text { infrage stellen von } \\
\text { Sachverhalten auf } \\
\text { der Basis vertiefter } \\
\text { inhaltlicher } \\
\text { Auseinandersetzung } \\
\end{array}$ & $\begin{array}{l}\text { Ich war viel mehr } \\
\text { bei der Sache als } \\
\text { bei einer Vorlesung, } \\
\text { ich habe mich } \\
\text { in die Materie } \\
\text { eingearbeitet. }\end{array}$ & 23 & 90 & 102 & 50 & 12 & 5 & $\begin{array}{c}\text { Ich habe nur die } \\
\text { Aufgaben abgearbeitet, } \\
\text { nur schnell alles } \\
\text { hinter mich gebracht, } \\
\text { eigentlich weiß ich } \\
\text { gar nicht, worum es } \\
\text { wirklich ging. }\end{array}$ & $\begin{array}{c}3 \\
2 ; 3\end{array}$ \\
\hline & $\begin{array}{l}\text { Effektive Teamarbeit } \\
\text { zeigen, im } \\
\text { interprofessionellen } \\
\text { Team argumentieren, } \\
\text { überzeugen, widerlegen }\end{array}$ & $\begin{array}{l}\text { Wir hatten Spaß an } \\
\text { der Teamarbeit, wir } \\
\text { haben die Arbeit } \\
\text { gut aufgeteilt und } \\
\text { uns gegenseitig } \\
\text { unterstützt. } \\
\end{array}$ & 149 & 94 & 32 & 3 & 4 & 0 & $\begin{array}{l}\text { Ich hätte das lieber } \\
\text { alleine gemacht, es } \\
\text { war echt mühsam, } \\
\text { wir konnten uns nicht } \\
\text { einigen. }\end{array}$ & $\begin{array}{c}1 \\
1 ; 2\end{array}$ \\
\hline \multirow[b]{2}{*}{$\begin{array}{c}4 \\
\text { Wertordnung }\end{array}$} & $\begin{array}{c}\text { Rollenverständnis } \\
\text { entwickeln, im Rahmen } \\
\text { einer Präsentation und } \\
\text { Diskussion verteidigen, } \\
\text { formulieren, verändern, } \\
\text { priorisieren }\end{array}$ & $\begin{array}{c}\text { Hat Spaß gemacht, } \\
\text { die Rollen einfach mal } \\
\text { auszuprobieren, habe } \\
\text { ein paar wertvolle } \\
\text { Erfahrungen } \\
\text { mitgenommen. } \\
\end{array}$ & 84 & 110 & 59 & 20 & 8 & 1 & $\begin{array}{l}\text { Absolut überflüssig, } \\
\text { dabei habe ich gar } \\
\text { nichts gelernt, was soll } \\
\text { das überhaupt? }\end{array}$ & $\begin{array}{c}2 \\
1 ; 3\end{array}$ \\
\hline & $\begin{array}{l}\text { Werte festlegen und } \\
\text { organisieren, das } \\
\text { interprofessionelle } \\
\text { Berufsfeld der } \\
\text { Arbeitsmedizin erfassen }\end{array}$ & $\begin{array}{l}\text { Ich habe eine } \\
\text { Vorstellung } \\
\text { davon, was } \\
\text { Physiotherapeuten/- } \\
\text { innen in der } \\
\text { Arbeitsmedizin } \\
\text { machen und wie } \\
\text { man so ein Angebot } \\
\text { angeht. }\end{array}$ & 80 & 117 & 73 & 11 & 1 & 0 & $\begin{array}{c}\text { Ich habe gar nichts } \\
\text { dazugelernt, keine } \\
\text { Ahnung was man als } \\
\text { Physiotherapeut/-in in } \\
\text { der Arbeitsmedizin so } \\
\text { macht. }\end{array}$ & $\begin{array}{c}2 \\
1 ; 3\end{array}$ \\
\hline
\end{tabular}

Anmerkung: Resultate der Lernzielstufe 2 (Wertbeantwortung) korrespondieren nicht mit der 6-stufigen Likert Skala. 6\% der Studierenden gaben hierbei an "überfordert" zu sein, 91\% gaben an "genau richtig gefordert" zu sein und 3\% gaben an "unterfordert" zu sein.

Ergebnisse lassen keine Aussagen darüber zu, ob Blended Learning im Vergleich zur Face-to-Face-Lehre positive Langzeiteffekte erwarten lässt. Allerdings ist in den unteren Lernzielstufen nur unter 4\% der Varianz auf die Intervention zurückzuführen. Diese Ergebnisse bestärken die Aussagen von Peroz et al. (2009), welche Blended Learning und Face-to-Face-Lehre in Bezug auf die Wissensspeicherung als gleich effektiv beschreiben, bzw. Nordin \& Alias, 2013, die eine nicht eindeutige Evidenz für die Effektivität des Blended Learnings sehen.

Für die Lernzielstufe 3 sind die Unterschiede zwischen Blended-Learning- und Kontroll-Kohorte in Bezug auf den Langzeiteffekt der Lernergebnisse nicht signifikant, was erneut die Aussage von Peroz et al. (2009) bestätigt. In der höchsten betrachteten Lernzielstufe (Lernzielstufe 4) schneidet die Blended-Learning-Kohorte schlechter ab als die Kontroll-Kohorte, dieser Unterschied ist zu $11 \%$ durch die Intervention erklärbar.
Somit ergaben sich hinsichtlich der kognitiven Ergebnisse signifikante Unterschiede zugunsten beider Kohorten, wobei die Effektstärken schwach bis mittel ausgeprägt waren. Daher kann diesbezüglich keine allgemeine Empfehlung zugunsten des traditionellen Lehr-LernSettings oder des Blended Learnings abgeleitet werden. Eine Erklärung für das schlechtere Abschneiden der Blended-Learning-Kohorte bei den Lernergebnissen auf Lernzielstufe 4 wäre der Bezug zur fehlenden Begleitung der Studierenden während der OnlinePhase durch die Lehrveranstaltungsleitung: In dieser Lehrveranstaltungssequenz erhielten die Studierenden kein Feedback durch die Lehrveranstaltungsleitung, sondern ausschließlich durch andere Studierende. Es wäre also durchaus möglich, dass im Lernprozess Lösungsmuster von den Studierenden falsch eingelernt und nicht ausreichend korrigiert wurden. Die Korrektur allfälliger unzureichender Lösungsstrategien durch die 
Lehrveranstaltungsleitung erfolgte im Blended Learning erst zu einem späteren Zeitpunkt. Es könnte also sein, dass im Langzeitgedächtnis die ursprünglich eingelernten insuffizienten Lösungsansätze die erfolgten Korrekturen in ihrer Wirkung überdauern. Im Face-to-FaceVortrag der Kontroll-Kohorte war die jeweils richtige Lösungsstrategie zu den Fallbeispielen bereits enthalten. Setzt man die Ergebnisse zur Lernzielstufe 4 in Bezug zu den Bildungsbiografien der Studierenden, kann man einen weiteren Einflussfaktor auf die individuelle Nutzung von Lernangeboten geltend machen. Die Lernbiografien der Studierenden unterscheiden sich nicht nur aufgrund ihres unterschiedlichen Einstiegsalters zu Beginn des Studiums (Mindestalter 18 Jahre und keine Altersbeschränkung war für Blended-Learning- und Kontroll-Kohorte gleich), sondern auch in Bezug auf ihre Lernerfahrungen in der dem Studium vorangegangenen individuellen Bildungsbiografie. Der Umgang mit Blended Learning muss vielfach erst geübt werden. Wenn Studierende bis zur untersuchten Lehrveranstaltung vorwiegend nicht selbstorganisiert gelernt haben, ist der erste Kontakt mit aktiven Lernformen noch ungewohnt. Inwieweit die Studierenden bereits Übung mit dem Lehr-Lernsetting des Blended Learning beim Einstieg in das Studium hatten, war jedoch nicht Gegenstand der Untersuchung. Es könnte relevant für den Langzeiteffekt des Blended Learnings sein, wenn Studierende keine, wenig oder viel Erfahrung mit diesem Lehr-Lernsetting haben. Die Lehrveranstaltung, in der das Blended Learning untersucht wurde, ist im 2. Semester verankert. Wenn man bedenkt, dass das Lernumfeld des 1. und 2. Semesters vorwiegend aus Face-to-Face-Lehre besteht, kann davon ausgegangen werden, dass die Studierenden im Rahmen des Studiums noch keine oder nur sehr wenig Erfahrung mit einem von großer Autonomie geprägten Lernprozess aufweisen.

\section{Diskussion der Ergebnisse zur affektiven Leistungsdimension}

Die Studierenden, die an der Lehrveranstaltung mit Blended Learning teilgenommen hatten, schätzten die Auswirkungen auf ihre affektive Leistungsdimension durchwegs positiv ein (Median und 75\%-Quartile immer $\leq 3$ bei einer 6-teiligen Likert Skala). Vor dem Hintergrund eines interprofessionellen Arbeitsfeldes im Bereich der Arbeitsmedizin ist der affektiven Leistungsdimension, die auch das Verhalten im Team prägt, Bedeutung beizumessen. Hier zeigt sich ein positiver Effekt des Blended Learnings. Die Einschätzung des Nutzens von Blended Learning für die affektive Leistungsdimension kann allerdings teilweise davon beeinflusst sein, wie ausgeprägt die Reflexionsfähigkeit der Studierenden bezogen auf ihren eigenen Lernprozess zum Zeitpunkt der Untersuchung war. Dennoch lässt sich eine eindeutige
Tendenz der Einschätzung aus den Ergebnissen ableiten. Studierende zeigten Spaß am Arbeiten im Team und am Ausprobieren der verschiedenen Rollen. Positive Emotionen (wie in Fragen zur Lernzielstufe 3 und 4 untersucht) fördern den Lernprozess und den damit verbundenen Kompetenzerwerb (Gläser-Zikuda, Stuchlíková, \& Janík, 2013; Green \& Batool, 2017), was im Hinblick auf erste positive Erfahrungen mit Rollen im interprofessionellen Team für das Gelingen einer effizienten Teamarbeit im realen Berufsfeld später entscheidend sein kann. Etwas geringer war die positive Bewertung auf der Lernzielstufe 1 (Wertbeachtung) nach Krathwohl (CEDEFOP, 2009) sowie auch in einer Kategorie der Lernzielstufe 3 (Wertung) ausgeprägt: Klarheit der Aufgabenstellung und Tiefe der inhaltlichen Auseinandersetzung mit der Materie waren im Vergleich zwar überwiegend positiv, aber nicht so gut bewertet wie die oben beschriebenen Lernergebnisse der affektiven Leistungsdimension. Hier kann man einen ähnlichen Bezug zu den Vorerfahrungen mit Elementen des Blended Learnings herstellen wie bei den Betrachtungen zur kognitiven Leistungsdimension. Studierende mit wenig Erfahrung in autonomer vertiefter inhaltlicher Auseinandersetzung mit Lernmaterialien haben demnach geringere Kompetenz im Erlangen von Klarheit über Aufgaben. Die Gewohnheit, Aufgaben zu erledigen, die in der Face-to-Face-Lehre erläutert und begleitet werden, muss erst ersetzt werden durch mehrfach geübten Umgang mit eigenständigem Erfassen von Aufgaben und Anforderungen.

Bezogen auf die Lernzielstufe 2 (Wertbeantwortung) nach Krathwohl (CEDEFOP, 2009) kann davon ausgegangen werden, dass das Erfahren eigener Leistungsbereitschaft als Reaktion bzw. Antwort auf die Anforderung der Aufgabenstellung die Studierenden ermutigt, in zukünftigen Lernprozessen und später auch im Berufsfeld auf bereits erprobte Lösungsstrategien zurückzugreifen und gestellten Anforderungen mit Leistungsbereitschaft zu begegnen.

\section{EINSCHRÄNKUNGEN}

Die vorliegenden Studienergebnisse sind als die einer beobachtenden Kohorten-Studie ohne experimentelles Design im Sinne einer Randomisierung oder BlockRandomisierung zu verstehen und $\mathrm{zu}$ interpretieren. Untersucht man den Langzeiteffekt von Lernergebnissen, muss man die Resultate in Bezug auf eine Reihe von nicht-isolierbaren Einflussfaktoren kritisch betrachten. Lernergebnisse, die unmittelbar nach Abschluss des Lernprozesses überprüft werden, unterliegen in der Regel kaum weiteren direkten Einflussfaktoren. Liegen aber wie bei der vorliegenden Studie zwischen Lernprozess und Überprüfung der Lernergebnisse 1,5 
Jahre und gibt es keine Ergebnisse unmittelbar nach der Lehrveranstaltung, kann davon ausgegangen werden, dass das ursprüngliche Lernergebnis durch eine Reihe von Einflussfaktoren verändert wurde: Studierende erweitern ihren Erfahrungshorizont laufend durch stattfindende Lernprozesse, die zwar nicht unmittelbar auf dieselbe Thematik abzielen, aber durch erwünschte Verknüpfungen jedenfalls das Themenfeld bereits abgeschlossener Lehrveranstaltungen berühren. Die Vernetzung von Lernergebnissen, die in aufeinander aufbauenden Lehrveranstaltungen angestrebt wird, trägt demnach dazu bei, dass Studierende Lernergebnisse in ihren individuellen Kontext einbetten. Es darf aber auch nicht übersehen werden, dass gerade bei einem Lernprozess, der zur Berufsqualifikation führen soll, der Langzeiteffekt von Lernergebnissen relevant für einen erfolgreichen Berufseinstieg ist. Die nicht-isolierbaren Einflussfaktoren wirken jedoch in der vorliegenden Untersuchung auf Kontroll- und Blended-LearningKohorte, wodurch die Auswertung der Daten nicht beeinträchtigt sein sollte.

Der in der Diskussion bereits angeführte Umstand, dass Studierende mit unterschiedlichen Lernbiografien auch unterschiedliche Vorerfahrungen für das Blended Learning mitbringen, ist als weitere Einschränkung der Ergebnisse anzuführen. In einer vertieften Untersuchung müsste auf diese Limitation insofern eingegangen werden, als man die untersuchten Populationen nach ihren Vorerfahrungen in Gruppen einteilen und damit den Bezug zu einem weiteren möglichen Einflussfaktor auf Lernergebnisse herstellen könnte.

Die affektive Leistungsdimension wurde ohne KontrollKohorte untersucht. Dadurch fehlt der Aspekt, inwiefern sich die Face-to-Face-Lehre im Vergleich zum Blended Learning auf die affektive Leistungsdimension auswirkt. Die Resultate der affektiven Leistungsdimension würden einen erweiterten Interpretationsrahmen erlauben, wenn die Untersuchung eine Kontroll-Kohorte einschließen würde.

\section{SCHLUSSFOLGERUNG}

In den unteren Lernzielstufen der kognitiven Leistungsdimension kann keinem der beiden LehrLernsettings der Vorzug gegeben werden. Es kann der Schluss gezogen werden, dass der Einsatz von Blended Learning für die kognitive Leistungsdimension in der höheren Lernzielstufe der Face-to-Face-Lehre minimal unterlegen ist. Der Einsatz von Blended Learning kann aber für die Entwicklung der affektiven Leistungsdimension der Studierenden für alle untersuchten Lernzielstufen empfohlen werden.

Wenn für die Entwicklung der kognitiven Leistungsdimension auf mehreren Lernzielstufen im Verlauf des Studiums keinem Lehr-Lernsetting eindeutig der Vorzug gegeben werden kann, so könnten andere Entscheidungskriterien im Vordergrund stehen. Lehrende könnten sich bei der Auswahl von LehrLernsettings am Constructive Alignment orientieren, welches lernergebnisorientierte Lehr- und Lernaktivitäten in den Vordergrund der Hochschullehre stellt (Tam, 2014). Im Weiteren wird die Auswahl aber auch vom individuellen Methoden-Pool der Lehrenden und den infrastrukturellen Rahmenbedingungen beeinflusst sein. Im Sinne der Studierendenzentriertheit von Lehre sollen die Erwartungshaltungen, die Vorerfahrungen und die Bedürfnisse der Studierenden bei der Auswahl der Lehrmethode in Betracht gezogen werden (European Association for Quality Assurance in Higher Education, 2015). Werden die Ergebnisse zur affektiven Leistungsdimension berücksichtigt, soll dem Einsatz von Blended Learning der Vorzug gegeben werden, wenn die im Kompetenzerwerb der Lehrveranstaltung angeführten Lernergebnisse zusätzlich zur kognitiven Leistungsdimension einen hohen Anteil an der affektiven Leistungsdimension aufweisen.

Voraussetzung für den Einsatz von Blended Learning ist darüber hinaus eine entsprechende Qualifikation der Lehrperson in der Anwendung des Blended Learnings und die infrastrukturelle Unterstützung des Blended Learnings im jeweiligen hochschulischen Rahmen. Entspricht der Einsatz von Blended Learning der Erwartungshaltung der Studierenden bzw. sind schon Erfahrungen mit der Lernumgebung des Blended Learning vorhanden, wird dies zum Erreichen der angestrebten Lernergebnisse zusätzlich beitragen.

In der vorliegenden Studie wurde nicht auf die individuellen Bildungsbiografien und Leistungsniveaus der Studierenden eingegangen. Dies könnte aber einen Einfluss auf die Effektivität von Blended Learning haben und könnte daher Gegenstand weiterführender Studien sein.

In der vorliegenden Studie blieb die psychomotorische Leistungsdimension (Schewior-Popp, 2014) ausgeklammert. Für Lehrende, die mit ihrer Lehrveranstaltung Lernergebnisse der psychomotorischen Leistungsdimension erzielen wollen, wäre eine Studie zum Effekt von Blended Learning, ebenfalls differenziert nach Lernzielstufen innerhalb dieser Leistungsdimension, eine Orientierungshilfe.

\section{DANKSAGUNG}

Die vorliegende Studie wurde am Bachelorstudiengang Physiotherapie durchgeführt und entspricht als Beitrag zur Umsetzung von studierendenzentriertem Lehren und Lernen dem Code of Conduct an der FH Campus Wien. Die Sequenzen des Blended Learnings 
wurden mit Unterstützung des Teaching Support Centers der FH Campus Wien entwickelt und von den Lehrveranstaltungsleiterinnen der Lehrveranstaltung „Arbeitsmedizin und Prävention“ umgesetzt. Der Start des Projekts wurde von Emalie Hurkmans, PhD begleitet.
Großer Dank gilt den Studierenden der beteiligten Jahrgänge, die sich für die Einschätzung des Nutzens von Blended Learning bzw. für die Untersuchung der Lernergebnisse im 6. Semester zur Verfügung gestellt haben.

\section{References}

Anderson, L. W., \& Krathwohl, D. R. (Hrsg.) (2001). A Taxonomy for Learning, Teaching, and Assessing. A Revision of Bloom's Taxonomy of Educational Objectives. New York: AddisonWesley.

Babb, S., Stewart, C., \& Johnson, R. (2010). Constructing Communication in blended Learning Environments: Students'Perception of Good Practice in Hybrid Courses. MERLOT Journal of Online Learning and Teaching, 6 (4), 735-753. Zugänglich unter: http://jolt.merlot. org/vol6no4/babb_1210.pdf

Bloom, B. S. (Hrsg.) (1972). Taxonomie von Lernzielen im kognitiven Bereich (4. Auflage). Weinheim und Basel: Beltz-Verlag.

Botezatu, M., Hult, H., Tessma, M. K., \& Fours, U. (2010). Virtual patient simulation: Knowledge gai or knowledge loss? Medical Teacher, 32(7), 562-568.

Boye, S., Moen, T., \& Vik, T. (2012). An e-learning course in medical immunology: Does it improve learning outcome? Medical Teacher, 34(9), e649-e653. Zugänglich unter: http://dx.doi.org/10.3109/014 2159X.2012.675456

Bruff, D. O., Fisher, D. H., McEwen K.E., \& Smith, B.E. (2013). Wrapping a MOOC:Student Perceptions of an Experiment in Blended Learning. MERLOT Journal of Online Learning and Teaching, 9(2), 187-199. Zugänglich unter: http://jolt.merlot.org/ vo19no2/bruff_0613.pdf

CEDEFOP, (2009). Der Perspektivwechsel hin zu Lernergebnissen PolitikundPraxis inEuropa. EuropäischesZentrumfürdieFörderung der Berufsbildung. Luxemburg: Amt für Veröffentlichungen der Europäischen Union. Zugänglich unter: http://erwachsenenbildung. at/themen/nqr/grundlagen/grundbegriffe.php\#lernergebnisse

Dziuban, C. D., Hartmann, J. L., \& Moskal, P. D. (2004). Blended Learning. EDUCAUSE Center for Applied Research Bulletin 2004(7), 1-44. Zugänglich unter: https://net.educause.edu/ir/ library/pdf/erb0407.pdf

Ebner, M., Schön, S., \& Nagler, W. (2013). Einführung. Das Themenfeld „Lernen und Lehren mit Technologien“. In: Ebner, M. \& Schön, S. (Hrsg.), L3T Lehrbuch für Lehren und Lernen mit Technologien. Zugänglich unter: http://creativecommons.org/licenses/by-sa/3.0/de/

Ellaway, R., \& Masters, K. (2008a). AMEE Guide 32: e-learning in medical education Part 1: Learning, teaching and assessment. Medical Teacher, 30(5), 455-473.

Ellaway, R., \& Masters, K. (2008b). E-learning in medical education Guide 32 Part2: Technology, management and design. Medical Teacher, 30(5), 474-489.

European Association for Quality Assurance in Higher Education, European Students' Union, European University Association \& European Association of Institutions in Higher Education, (2015). Standards and Guidelines for Quality Assurance in the European Higher Education Area (ESG). Brüssel: EURASHE. Zugänglich unter: http://www.enqa.eu/wp-content/uploads/2015/11/ ESG_2015.pdf

Field, A. (2013). Discovering Statistics Using IBM SPSS Statistics. (4. Ausgabe). Los Angeles: Sage Publications.

Garnham, C., \& Kaleta, R. (2002). Introduction to Hybrid Courses. Teaching with Technology Today, 8(6). Zugänglich unter: https:// hccelearning.files.wordpress.com/2010/09/introduction-to-hybridcourse1.pdf

Gläser-Zikuda, M., Stuchlíková, I., \& Janík, T. (2013). Emotional Aspects of Learningand Teaching: Reviewing the Field Discussing the Issues. Orbis Scholae, 7(2), 7-22. Zugänglich unter: http://www.orbisscholae.cz/archiv/2013/2013_2_01.pdf

Gormley, G. J., Collins, K., Boohan, M., Bickle, I. C., \& Stevenson M. (2009). Is there a place for e-learning in clinical skills? A survey of undergraduate medical students' experiences and attitudes. Medical Teacher, 31(1), e6-e12. Zugänglich unter: http://dx.doi. org/10.1080/01421590802334317

Graham, C. R. (2006). Blended Learning Systems. In: Bonk, C. J. (Hrsg.), The handbook of blended learning: global perspectives, local designs (S. 3-19). San Francisco: Pfeiffer.

Green, Z., A., \& Batool, S. (2017). Emotionalized learning experiences: Tapping into the affective domain. Evaluation and Program Planning, 62(2017) 35-48. Zugänglich unter: http://dx.doi. org/10.1016/j.evalprogplan.2017.02.004

Güzer, B., \& Caner, H. (2014). The past, present and future of blended learning: an in depth analysis of literature. Procedia Social and Behavioral Sciences, 116(2014), 4596-4603. Zugänglich unter: http://dx.doi.org/10.1016/j.sbspro.2014.01.992

Ilic, D., Nordin, R. B., Glasziou, P., Tilson, J. K., \& Villanueva, E. (2015). A randomised controlled trial of a blended learning education interventionfor teaching evidence-based medicine. BMC Medical Education, 15(39), 1-10. Zugänglich unter: http:// bmcmededuc.biomedcentral.com/articles/10.1186/s12909-0150321-6

Ivarsson A., Anderson M. B., Johnson U., \& Lindwall M. (2013). To adjust or not adjust: Nonparametric effect sizes, confidence intervals, and real-world meaning. Psychology of Sport and Exercise, 14(2013), 97-102.

Kaur, M. (2013). Blended learning - its challenges and future. Procedia Social and Behavioral Sciences, 93(2013), 612-617. Zugänglich unter: http://dx.doi.org/10.1016/j.sbspro.2013.09.248

Kerres, M., \& de Witt, C. (2004). Pragmatismus als theoretische Grundlage für die Konzeption von eLearning. In: Mayer, H. O., Treichel, D. (Hrsg.), Handlungsorientiertes Lernen und eLearning (S.78-99). München: Oldenbourg-Verlag.

Koraneekij, P., \& Khlaisang, J. (2015). Development of Learning Outcome Based E-Portfolio Model Emphasizing on Cognitive Skills 
in Pedagogical Blended E-Learning Environment for Undergratuate Students at Faculty of Education, Chulalongkorn University. Procedia Social and Behavioral Sciences, 174(2015), 805-813. Zugänglich unter: http://dx.doi.org/10.1016/j.sbspro.2015.01.664

Krathwohl, D. R. (2002). A Revision of Bloom's Taxonomy: An Overview. Theory Into Practice, 41(4), 212-218.

Krathwohl, D. R., Bloom, B. S., \& Bertram, B. M. (1973). Taxonomy of Educational Objectives, the Classification of Educational Goals. Handbook II: Affective Domain. New York: David McKay Co. Inc.

Kröncke, K.-D. (2010). Computer-based learning versus practical course in pre-clinical education: Acceptance and knowledge retention. Medical Teacher, 32(5), 408-413.

Makhdoom, N., Khoshhal, K.I., Algaidi, S., Heissam, K., \& Zolaly, M. A. (2012). 'Blended learning' as an effective teaching and learning strategy in clinical medicine: a comparative cross-sectional university-based study. Journal of Taibah University Medical Sciences, 8 (1), 12-17.

Mayer, O., \& Kriz, W. (2010). Evaluation von eLernprozessen. München: Oldenbourg-Verlag.

Means, B., Toyama, Y., Murphy, R., Bakia, M., \& Jones, K. (2010). Evaluation of Evidence-Based Pratices in Online Learning: A Meta-Analysis and Review of Online Learning Studies. Washington, D.C.: U. S. Department of Education, Office of Planning, Evaluation, and Policy Development. Zugänglich unter: http://repository.alt.ac.uk/629/

Morton, C. E., Saleh, S. N., Smith, F. S., Hemani, A., Ameen, A., Bennie, T. D., \& Toro-Troconis, M. (2016). Blended learning: how can we optimise undergraduate student engagement? BMC Medical Education, 16(195), 1-8. Zugänglich unter: https://www.ncbi.nlm. nih.gov/pmc/articles/PMC4973547/pdf/12909_2016_Article_716. pdf

Nordin, A. B., \& Alias, N. (2013). Learning Outcomes and Student Perception in Using of Blended Learning in History. Procedia Social and Behavioral Sciences, 103(2013), 577-585.

Owston, R., York, D., \& Murtha, S. (2013). Student perceptions and achievement in a university blended learning strategic initiative. The Internet and Higher Education, 18, 38-46. Zugänglich unter: http://dx.doi.org/10.1016/j.iheduc.2012.12.003

Peroz, I., Beuche, A., \& Persoz, N. (2009). Randomized controlled trial comparing lecture versus self studying by an online tool. Medical Teacher, 31(6), 508-512.
Physio Austria (Hrsg.) (2016). Die Physiotherapeutin/Der Physiotherapeut. Kompetenzprofil. Wien: Physio Austria Bundesverband der PhysiotherapeutInnen Österreichs. Zugänglich unter: http://www.physioaustria.at/system/files/general/phy_ kompetenzprofil_deutsch_fin_022016.pdf

Poonam, K. \& Prajna, P. S. (2016). Blended learning approach for teaching and learning anatomy: Students`and teachers ’erspective. Journal of the Anatomical Society of India, 65(1), 43-47. Zugänglich unter: http://dx.doi.org/10.1016/j.jasi.2016.06.001

Reasons, S., Valadares, K., \& Slavkin, M. (2005). Questioning the hybrid model: Student outcomes in different course formats. Journal of Asynchronous Learning, 9(1), 83-94.

River, J., Currie, J., Crawford, T., Betihavas V., \& Randall, S. (2016). A systematic review examining the effectiveness of blended technology with team-based learning. Nurse Education Today, 45(2016), 185-192. Zugänglich unter: http://dx.doi.org/10.1016/j. nedt.2016.08.012

Rowe, M., Frantz, J., \& Bozalek, V. (2012). The role of blended learning in the clinical education of healthcare students: A systematic review. Medical Teacher, 34(4), e216-e221. Zugänglich unter: http://www. tandfonline.com/doi/full/10.3109/0142159X.2012.642831

Ruiz, J. G., Mintzer, M. J., \& Leipzig, R. M. (2006). The Impact of E-Learning in Medical Education. Academic Medicine, 81(3), 207-212.

Schewior-Popp, S. (2014). Lernsituationen planen und gestalten. Handlungsorientierter Unterricht im Lernfeldkontext (2. Auflage). Stuttgart und New York: Georg-Thieme-Verlag.

Tam, M. (2014). Outcomes-based approach to quality assessment and curriculum improvement in higher education. Quality Assurance in Education, 22(2), e158-e168. Zugänglich unter: http://www. emeraldinsight.com/doi/pdfplus/10.1108/QAE-09-2011-0059

Tomczak, M., \& Tomczak, E. (2014). The need to report effect size estimates revisited. An overview of some recommended measures of effect size. Trends in Sport Sciences, 1(21), 19-25.

Tosun, S. (2015). The effects of blended learning on EFL students'vocabulary enhancement. Procedia Social and Behavioral Sciences, 199(2015), 641-647. Zugänglich unter: http://dx.doi. org/10.1016/j.sbspro.2015.07.592 